Supporting information

Bifunctional Electrocatalytic Activity of Ordered Intermetallics Based on Pd and Sn

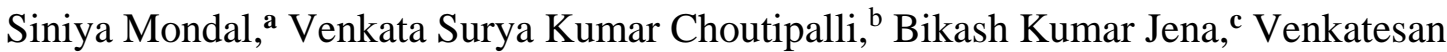 \\ Subramanian $^{\mathrm{b}}$ and C. Retna Raj*a
}

a Functional Materials and Electrochemistry Lab, Department of Chemistry Indian Institute of Technology, Kharagpur

Kharagpur 721302, India

E-mail: crraj@chem.iitkgp.ernet.in

b Chemical Laboratory, CSIR-Central Leather Research Institute, Adyar, Chennai 600020, India

c CSIR-Institute of Minerals and Materials Technology, Bhubaneswar

Odisha, India-751013 


\section{Synthesis of graphene oxide}

Graphene oxide (GO) was synthesized from natural graphite powder according to modified Hummer's method. In a typical procedure, $25 \mathrm{ml}$ concentrated $\mathrm{H}_{2} \mathrm{SO}_{4}$ was slowly added to a mixture of $0.5 \mathrm{~g}$ graphite powder and $0.5 \mathrm{~g} \mathrm{NaNO}_{3}$ in a $500 \mathrm{ml}$ round-bottomed flask maintaining $0{ }^{\circ} \mathrm{C}$ temperature under stirring condition. The mixture was continuously stirred while $2.5 \mathrm{~g}$ of solid $\mathrm{KMnO}_{4}$ was slowly poured to the reaction vessel. After that, ice-bath was removed and the mixture was stirred for another $4 \mathrm{~h}$ followed by addition of $150 \mathrm{ml}$ of Millipore water. Finally, $30 \% \mathrm{H}_{2} \mathrm{O}_{2}$ was added to the reaction mixture under stirring condition until the termination of gas effervescence. The unexfoliated graphite and oxidizing agents in the resulting mixture was removed by centrifugation. The residue was then washed repeatedly with $15 \% \mathrm{HCl}$ solution. The washing was continued until the filtrate gave a negative test for the presence of sulphate ions with $\mathrm{BaCl}_{2}$ solution. Finally, the collected product was washed with Millipore water and dried under vacuum. The brown colored GO was obtained thereafter. 
Figure S1. (A) FTIR profiles of GO, and PdSn/rGO hybrid materials before and after pyrolysis. (B) XRD profiles of Pd-Sn/rGO hybrid material before and after thermal annealing and $\mathrm{Pd} / \mathrm{rGO}$.
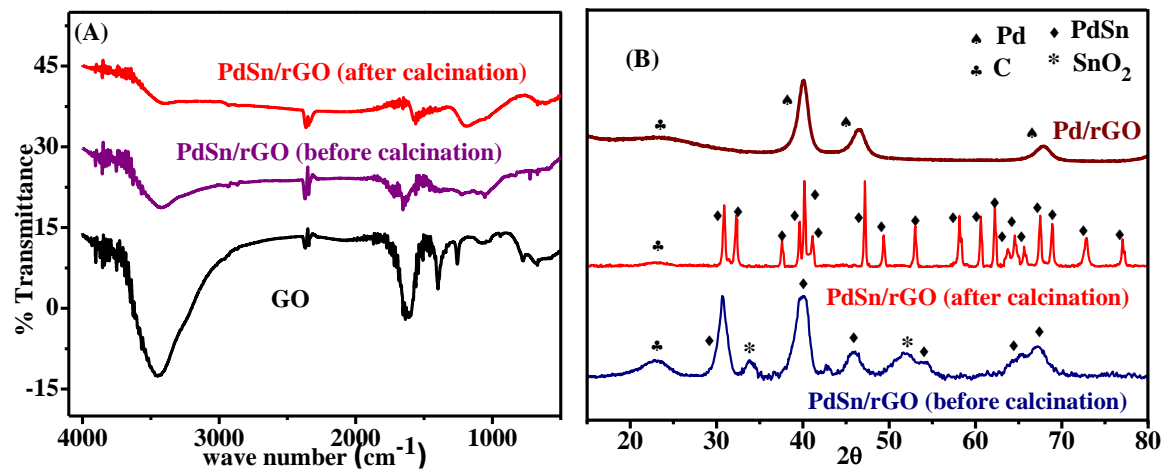
Figure S2. Raman spectra of GO and the hybrid intermetallics.

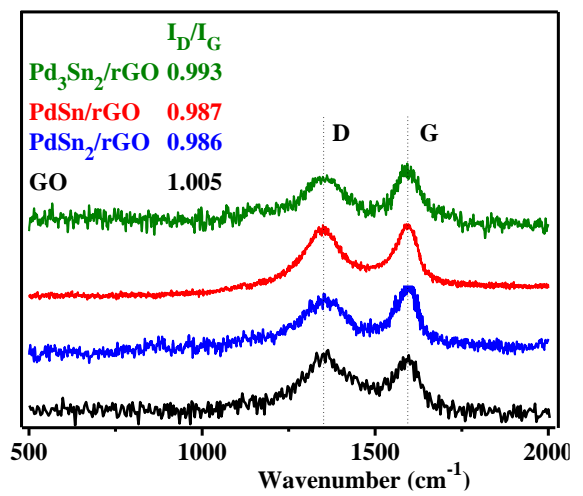


Figure S3. (A) XPS surface survey scan and (B) deconvoluted Pd 3d spectral profile of Pd/rGO hybrid catalyst.
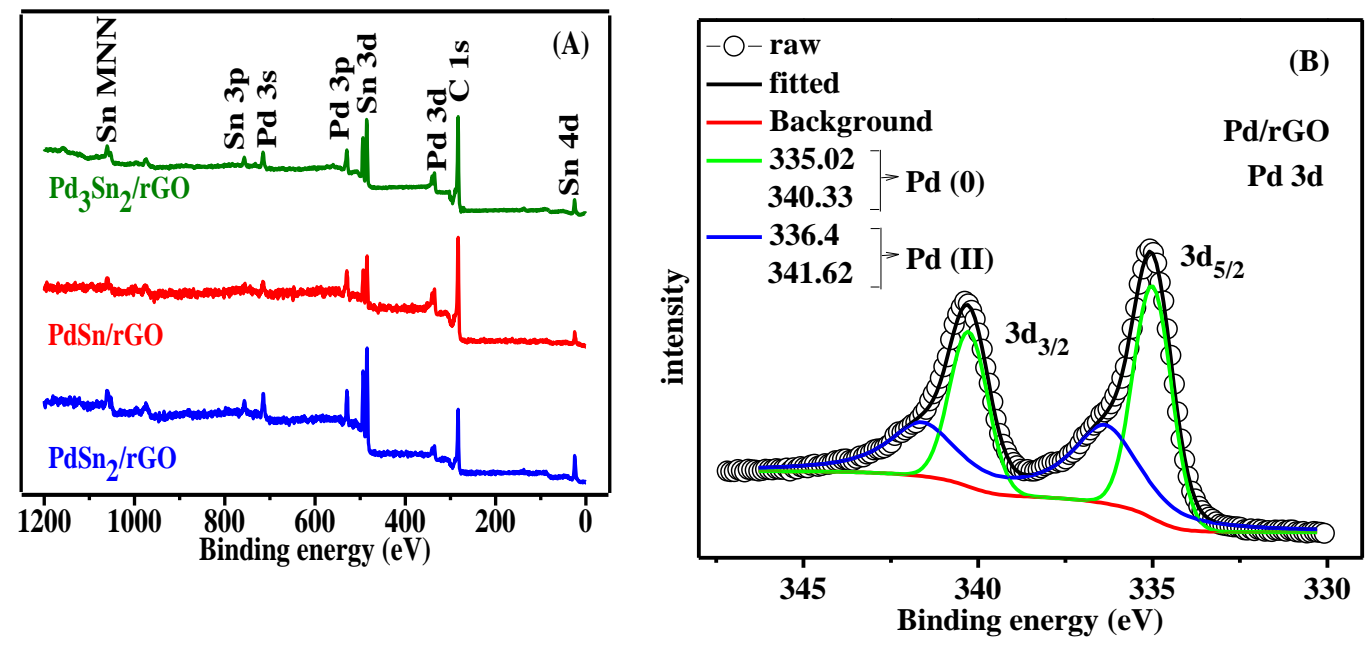
Figure S4. High resolution $\mathrm{Pd} 3 \mathrm{~d}(\mathrm{~A}-\mathrm{C})$ and $\mathrm{Sn} 3 \mathrm{~d}(\mathrm{D}-\mathrm{F})$ XPS profiles of unsupported $\mathrm{Pd}_{3} \mathrm{Sn}_{2}$ $(\mathrm{A}, \mathrm{D}), \mathrm{PdSn}(\mathrm{B}, \mathrm{E})$ and $\mathrm{PdSn}_{2}(\mathrm{C}, \mathrm{F})$ intermetallics.
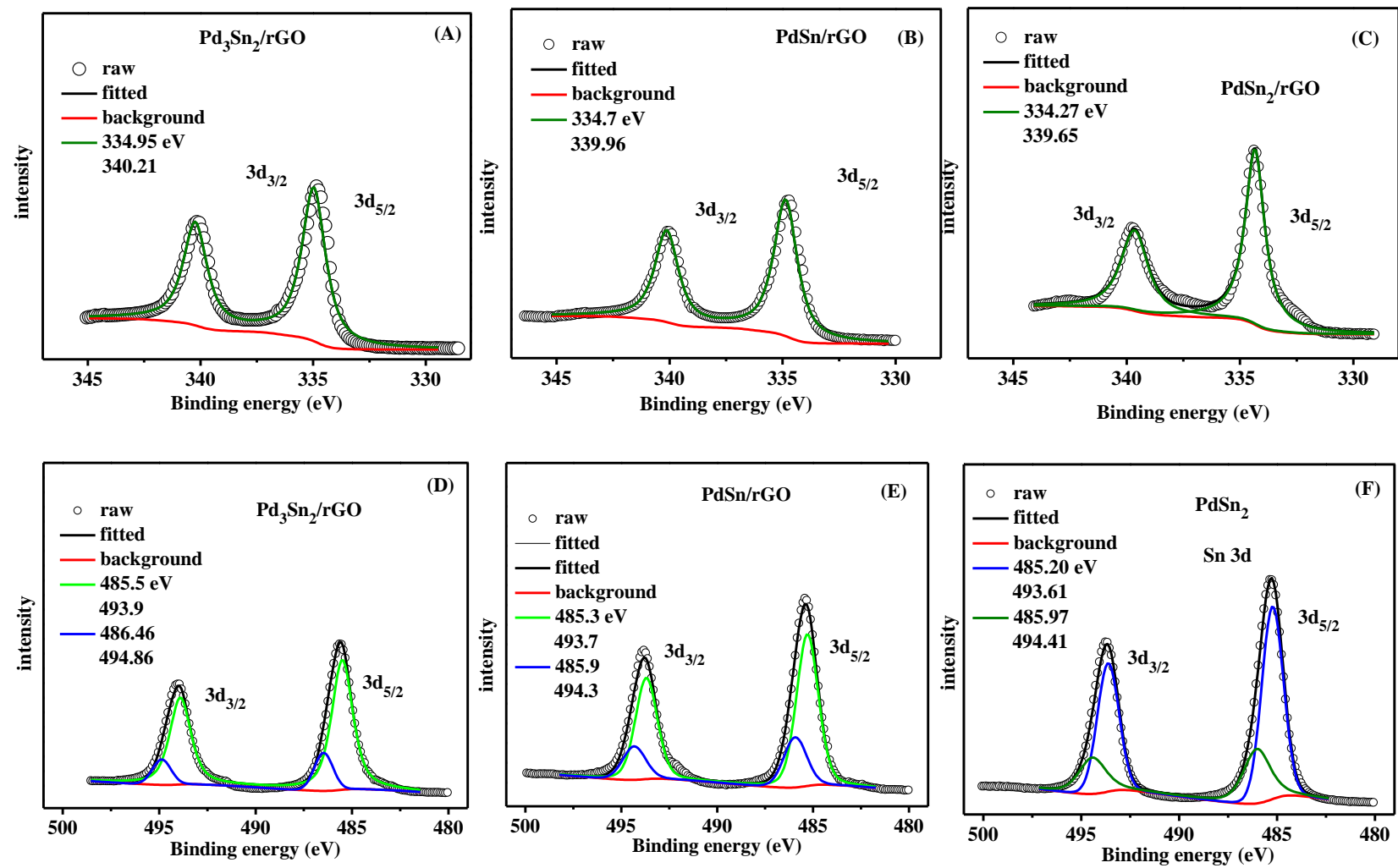
Figure S5. Deconvoluted Pd 3d (A-C) and Sn 3d (D-F) spectral profile of Pd-Sn/rGO hybrid intermetallics.
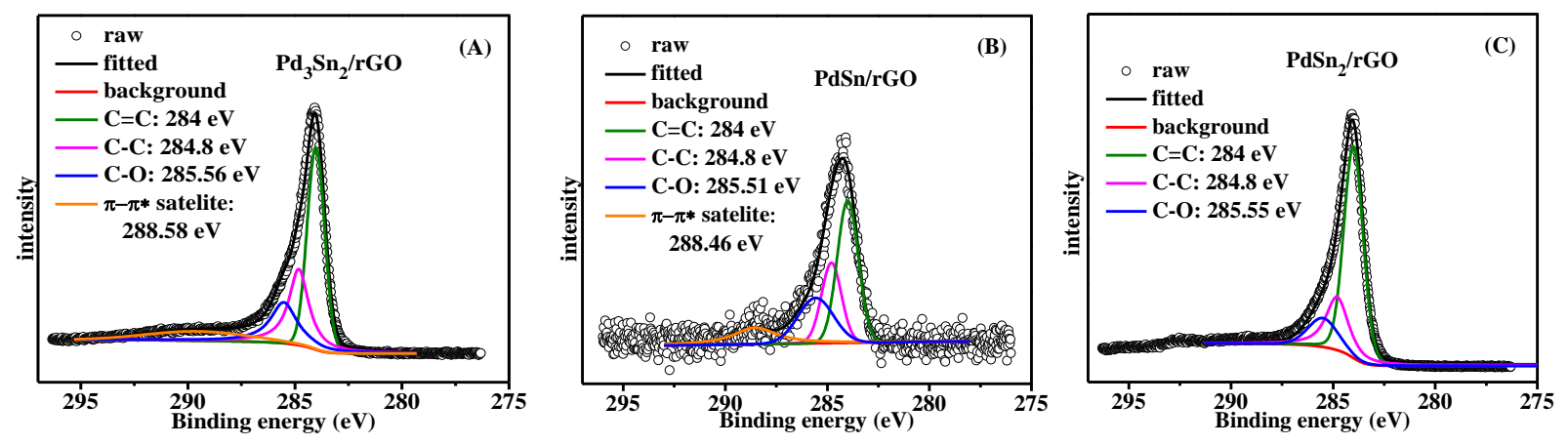
Figure S6. (A-B) Characteristic cyclic voltammograms obtained for as-synthesized hybrid intermetallics, $\mathrm{Pd} / \mathrm{rGO}$ hybrid material and $\mathrm{PdSn}$ in $0.1 \mathrm{M} \mathrm{KOH}$. (C-D) Linear sweep voltammetric profiles illustrating $\mathrm{CO}$ stripping $\mathrm{CO}$-saturated alkaline electrolyte. Scan rate: 50 $\mathrm{mV} / \mathrm{s}$.
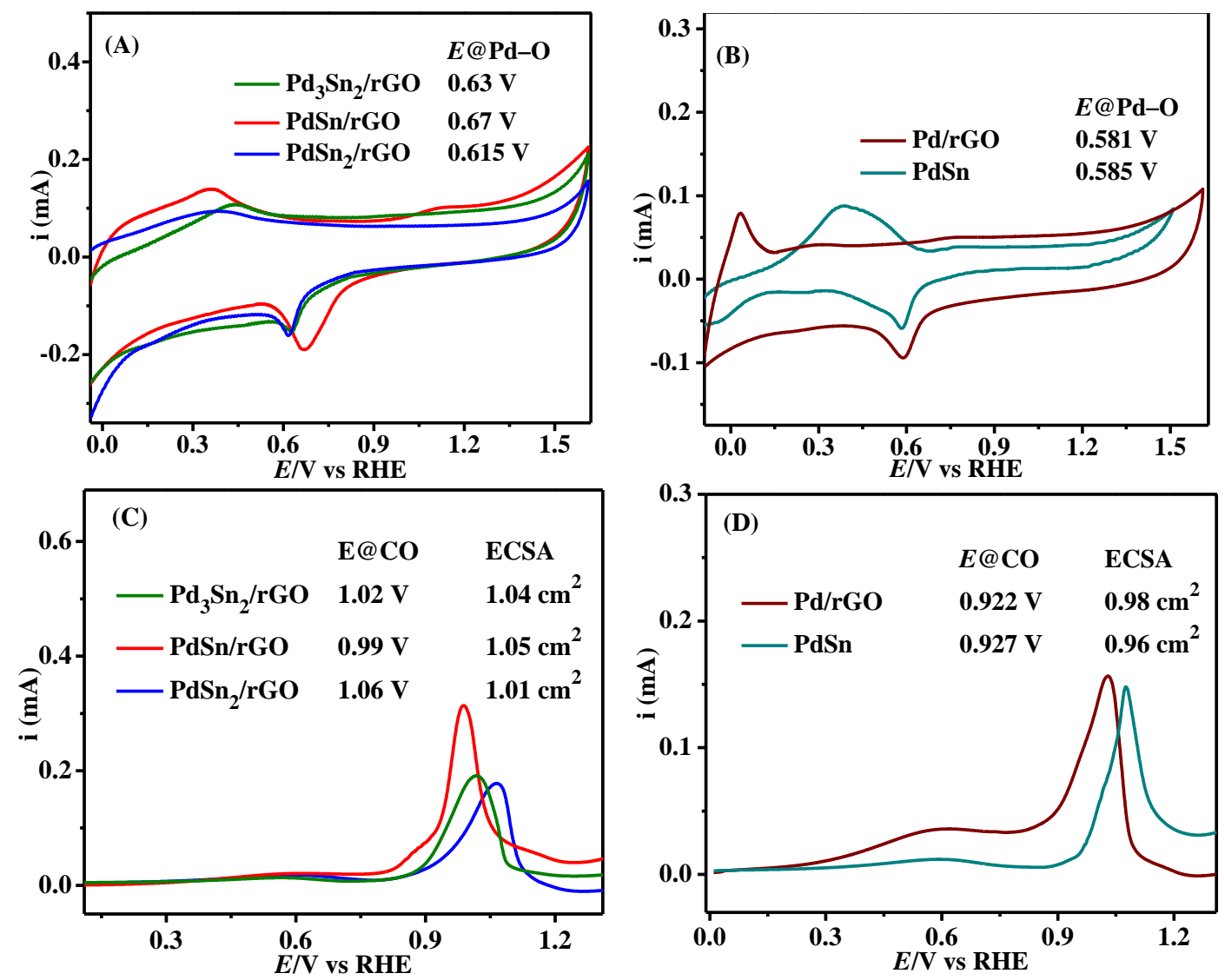

The cyclic voltammogram of $\mathrm{Pd}-\mathrm{Sn} / \mathrm{rGO}$ hybrid material shows the characteristic hydrogen adsorption/desorption signature for Pd. The peak corresponding to the surface oxide reduction $(E @ P d-O)$ is significant to understand the oxophilicity of the Pd active site. The less negative $E @ P d-O$ indicates decreased oxophilicity and weakly bound oxygenated species (eg. $O H_{a d s}$, $\left.O O H_{\text {ads }}\right)$ with Pd surface. 
Figure S7. Characteristic cyclic voltammograms obtained for (A) Pd-Sn/rGO and (B) Pd/rGO hybrid and $\mathrm{PdSn}$ intermetallics in $0.5 \mathrm{M} \mathrm{H}_{2} \mathrm{SO}_{4}$. Scan rate: $50 \mathrm{mV} / \mathrm{s}$.
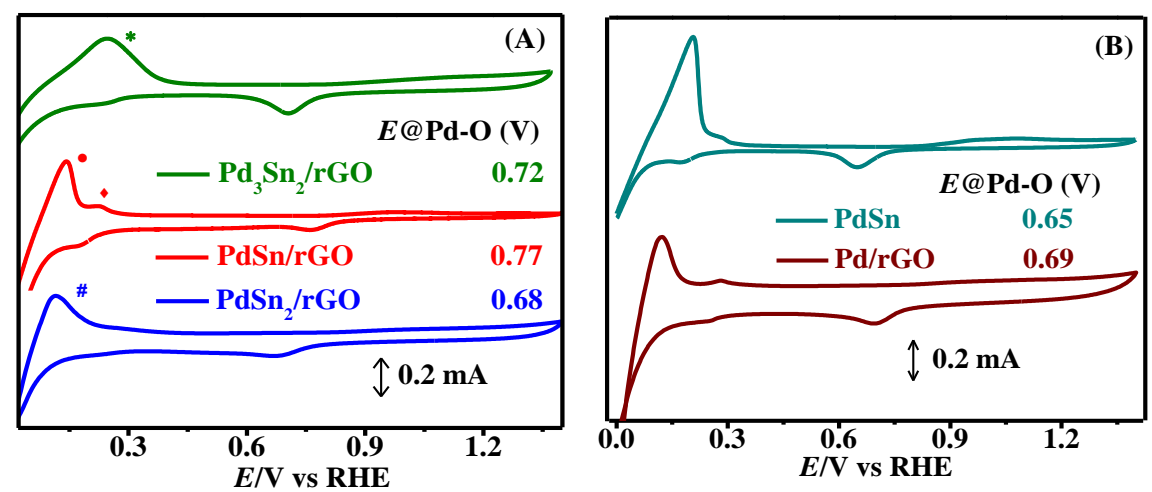

$\mathrm{Pd}-\mathrm{Sn} / \mathrm{rGO}$ hybrid materials show well-defined characteristic voltammetric signature in acidic electrolyte. The $\mathrm{Pd}_{3} \mathrm{Sn}_{2} / \mathrm{rGO}$ and $\mathrm{PdSn} / \mathrm{rGO}$ hybrid catalysts show a single peak (*, \#) at 0.25 and $0.12 \mathrm{~V}$, respectively, corresponding to the hydrogen desorption whereas $\mathrm{PdSn} / \mathrm{rGO}$ hybrid catalyst shows two distinct peaks $(\bullet, \bullet)$ at 0.14 and $0.224 \mathrm{~V}$ for the removal of adsorbed hydrogen on PdSn active site. These voltammetric features are very similar to that of Pd and can be ascribed to the removal of adsorbed hydrogen from the (111) and (200) planes of PdSn intermetallic compound. The hydrogen adsorption/desorption peaks of unsupported $\mathrm{PdSn}$ nanoparticles appeared at more positive potential (0.2 and $0.27 \mathrm{~V})$. 
Figure S8. Polarization curves obtained in RRDE analysis for ORR with hybrid intermetallics in $\mathrm{O}_{2}$ saturated $0.1 \mathrm{M} \mathrm{KOH}$. Scan rate: $5 \mathrm{mV} / \mathrm{s}$.

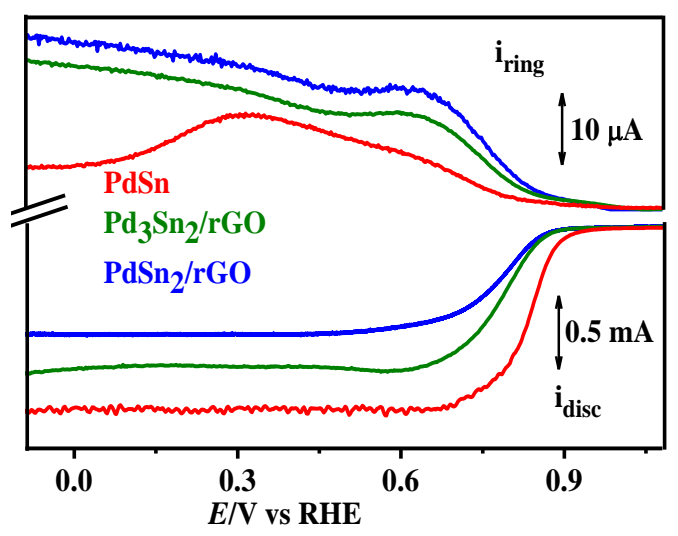


Figure S9. Proposed mechanistic pathway for electrochemical ORR and chemical disproportionation of $\mathrm{H}_{2} \mathrm{O}_{2}$. * and $b$ at the subscript denote the species absorbed on the electrode surface and in the bulk, respectively.

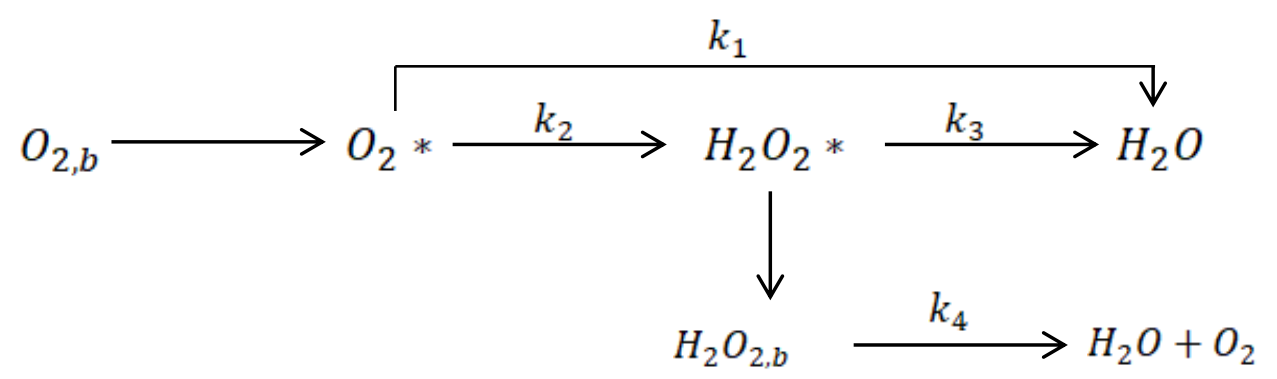

The rate constants associated with the ORR kinetics was analyzed according to Damjanovic. Two possible pathways have been proposed for the reduction of oxygen. In the parallel pathway, simultaneous 2- and 4-electron transfer occurs. The rate constant for direct reduction of oxygen is $k_{1}$. In the series pathway, oxygen is reduced to $\mathrm{H}_{2} \mathrm{O}\left(k_{2}, k_{3}\right)$ through the formation of intermediate $\mathrm{H}_{2} \mathrm{O}_{2}$. The rate constant for disproportionation of $\mathrm{H}_{2} \mathrm{O}_{2}$ is $k_{4}$. 
Figure S10. (A) Plot illustrating the durability of the catalysts towards ORR. Amperometric current obtained at the potential of $0.82 \mathrm{~V}$ is plotted against time. (B-D) Polarization curves obtained for ORR before and after amperometric durability test. The electrode was held at the potential of $0.82 \mathrm{~V}$ for $5 \mathrm{~h}$. Electrolyte: $\mathrm{O}_{2}$ saturated $0.1 \mathrm{M} \mathrm{KOH}$. Scan rate: $5 \mathrm{mV} / \mathrm{s}$.
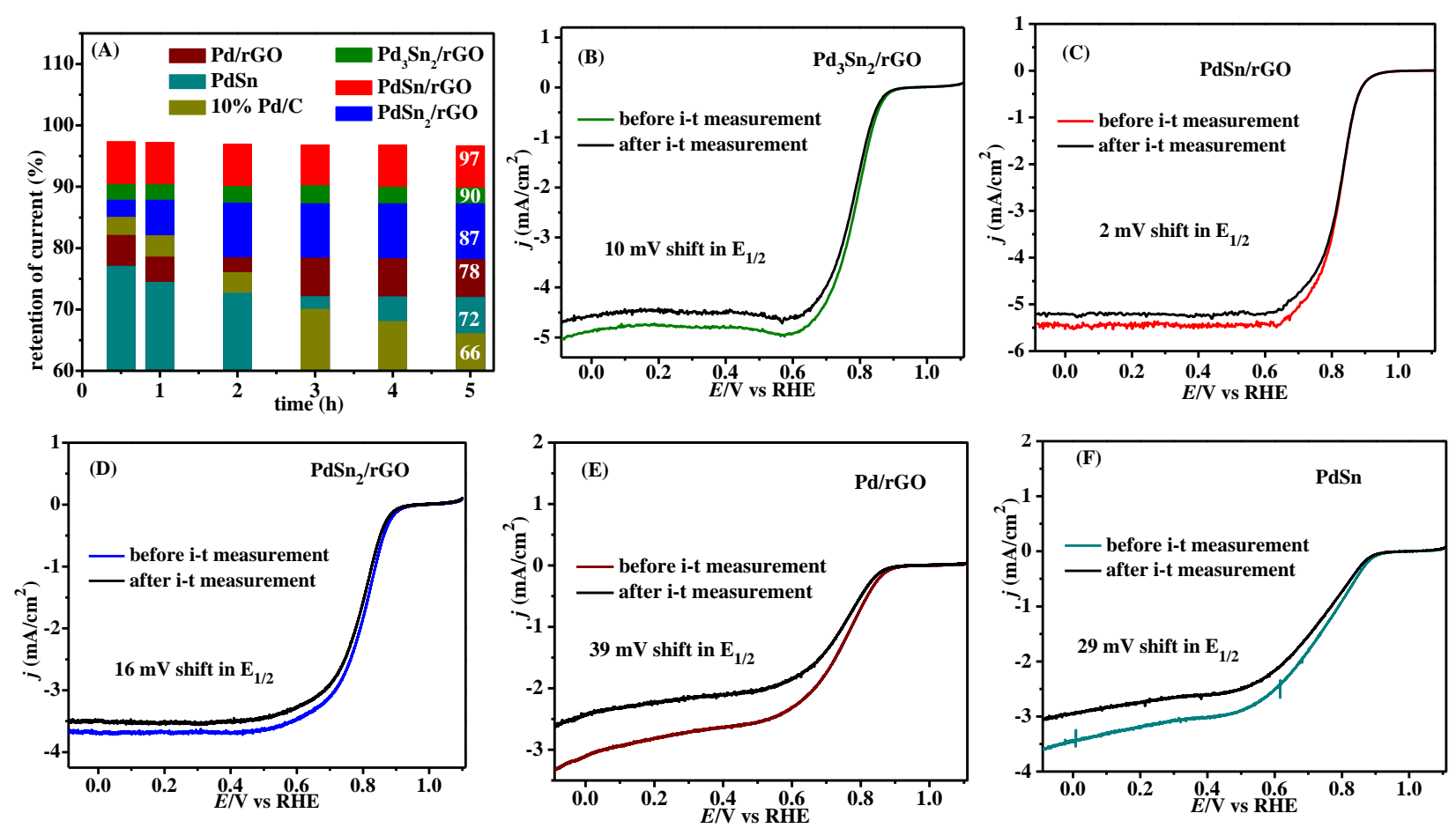
Figure S11. (A) Amperometric i-t curve illustrating the durability of the catalysts towards ORR in acidic condition. (B-D) Polarization curves obtained for ORR before and after the durability test. The potential was held at $0.88 \mathrm{~V}$ for $5 \mathrm{~h}$. Electrolyte: $\mathrm{O}_{2}$-saturated $0.5 \mathrm{M} \mathrm{H}_{2} \mathrm{SO}_{4}$. Scan rate: $5 \mathrm{mV} / \mathrm{s}$.
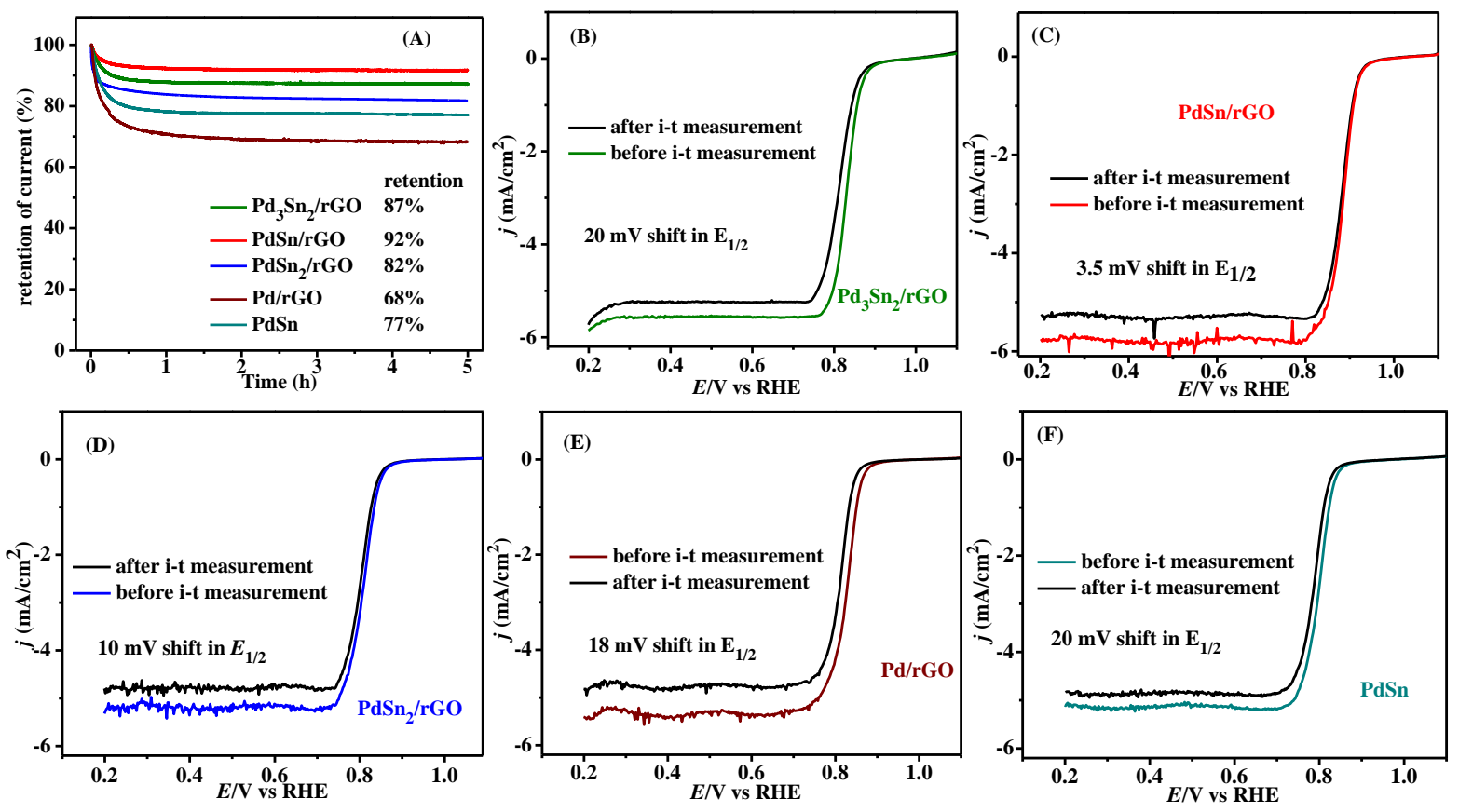
Figure S12. Polarization curves illustrating the durability of the hybrid material in alkaline (A) and acidic electrolyte (B) evaluated by registering polarization curves before and after continuous 5000 potential cycles. Scan rate: $5 \mathrm{mV} / \mathrm{s}$.
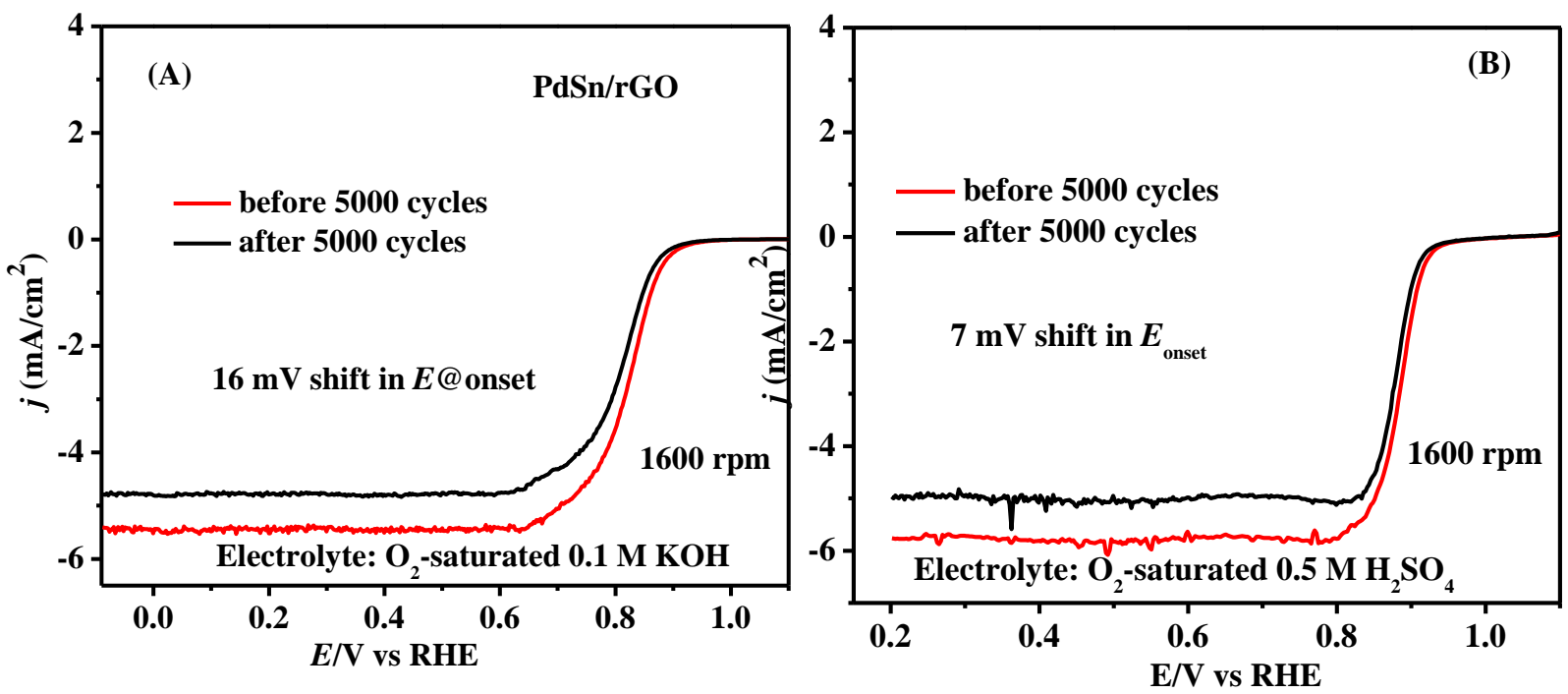
Figure S13. (A) Durability of the catalysts toward EGOR: Amperometric i-t curves obtained while the potential at $0.85 \mathrm{~V}$ for $3600 \mathrm{~s}$. (B-F) The voltammetric response of the catalysts towards EGOR before and after amperometric durability test. Electrolyte: $0.1 \mathrm{M} \mathrm{KOH}+0.5 \mathrm{M}$ ethylene glycol. Scan rate: $50 \mathrm{mV} \mathrm{s}^{-1}$.
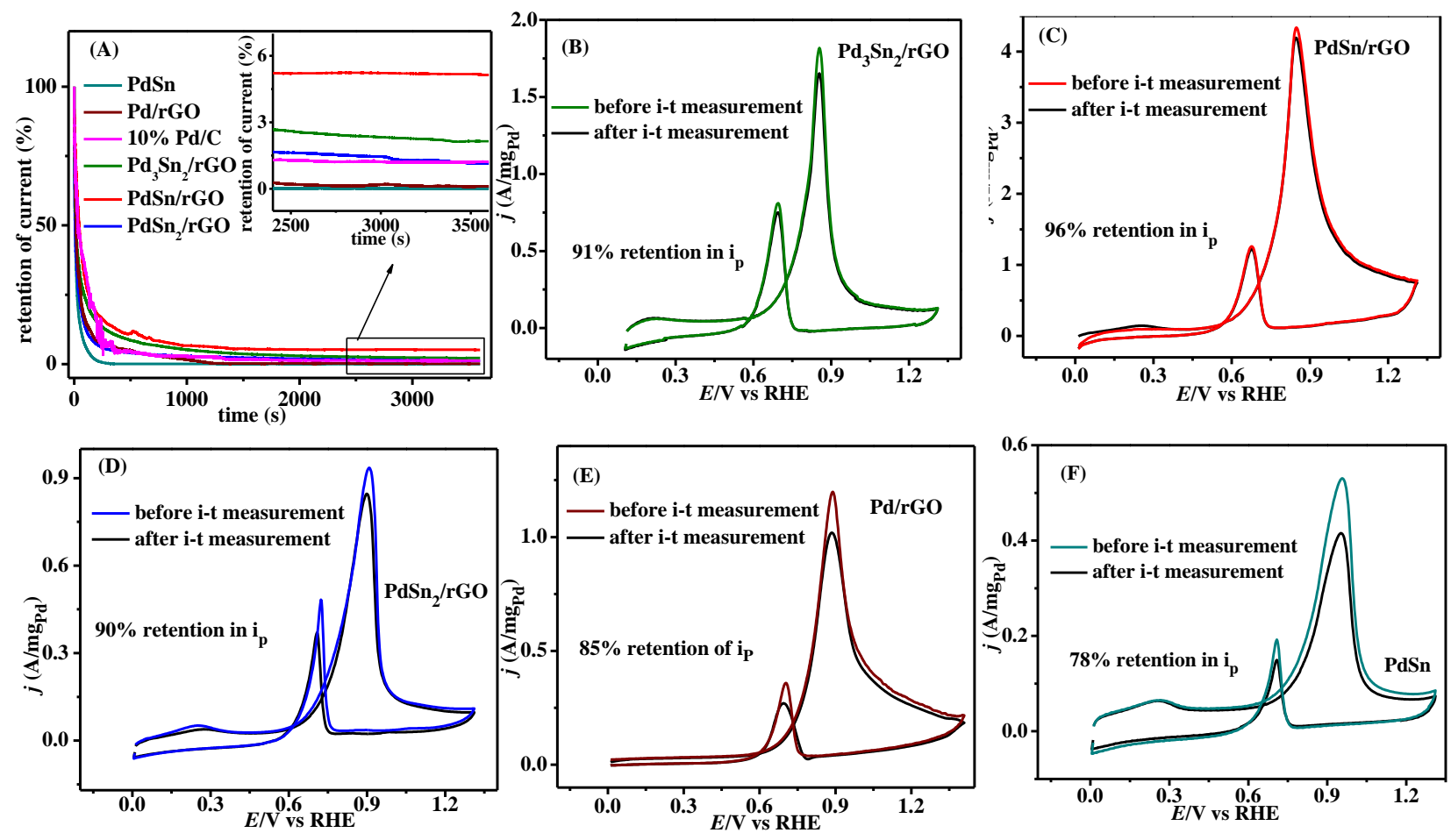
Figure S14. The crystal structures and the crystallographic data of $\mathrm{Pd}_{3} \mathrm{Sn}_{2}, \mathrm{PdSn}$ and $\mathrm{PdSn}_{2}$ intermetallics. (Data obtained from VESTA program)

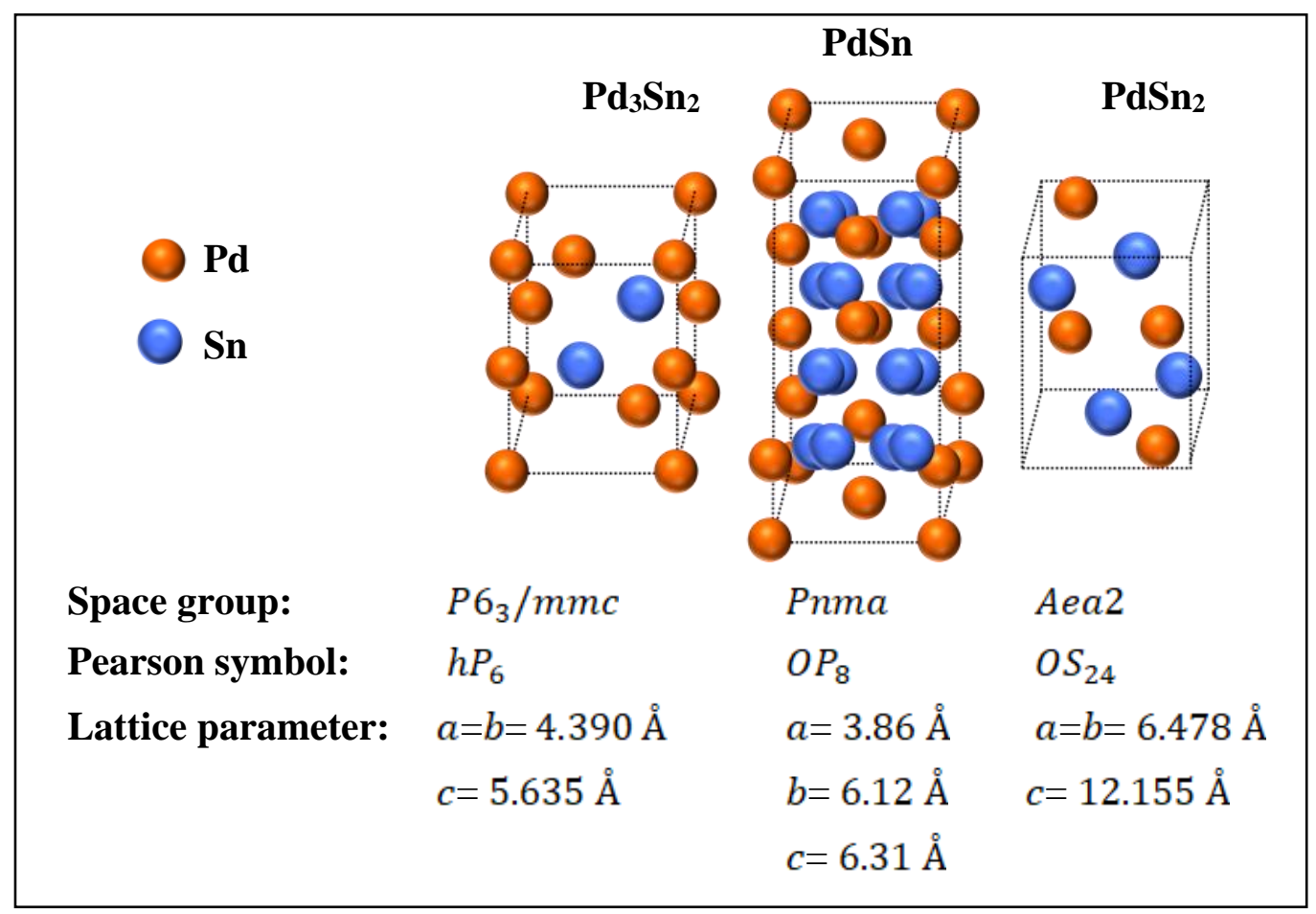


Table S1. Summary of the existing Pd and Sn based catalysts towards EGOR and ORR.

\begin{tabular}{|c|c|c|c|c|c|}
\hline \multicolumn{6}{|c|}{ EGOR } \\
\hline Catalysts & Electrolyte & $\begin{array}{c}\text { Scan rate } \\
(\mathrm{mV} / \mathrm{s})\end{array}$ & $\begin{array}{l}\text { Forward } \\
\text { peak } \\
\text { potential }\end{array}$ & Current density & References \\
\hline $\begin{array}{l}\text { PdSn on } \\
\text { sulfonated } \\
\text { MWCNT }\end{array}$ & $\begin{array}{l}0.5 \mathrm{M} \mathrm{EG}+ \\
0.5 \mathrm{M} \mathrm{KOH}\end{array}$ & 50 & $\begin{array}{l}-0.44 \mathrm{~V} \text { vs } \\
\mathrm{Ag} / \mathrm{AgCl}\end{array}$ & $51.9 \mathrm{~mA} / \mathrm{cm}^{2}$ & $\begin{array}{l}\text { J. Electroanal. } \\
\text { Chem. 2013, } \\
692,26-30\end{array}$ \\
\hline $\begin{array}{l}\mathrm{PdSn} / \mathrm{N} \text {-doped } \\
\text { Graphene (2:1 } \\
\text { molar ratio) }\end{array}$ & $\begin{array}{l}0.5 \mathrm{M} \mathrm{EG}+ \\
1.0 \mathrm{M} \mathrm{KOH}\end{array}$ & 50 & $\begin{array}{l}-0.03 \mathrm{~V} \text { vs } \\
\mathrm{Hg} / \mathrm{HgO}\end{array}$ & $118.05 \mathrm{~mA} / \mathrm{cm}^{2}$ & $\begin{array}{l}\text { Sci. Rep. 2015, } \\
5,14173 .\end{array}$ \\
\hline $\begin{array}{l}\text { PdSn/rGO } \\
\text { hybrid } \\
\text { intermetallics }\end{array}$ & $\begin{array}{l}0.5 \text { M EG } \\
+0.1 \mathrm{M} \\
\mathrm{KOH}\end{array}$ & 50 & $\begin{array}{l}\text { 0.848 V vs } \\
\text { RHE }\end{array}$ & $4.34 \mathrm{~A} / \mathrm{mg}_{\mathbf{P d}}$ & This Work \\
\hline \multicolumn{6}{|c|}{ ORR } \\
\hline Catalysts & Electrolyte & $\begin{array}{c}\text { Scan rate } \\
(\mathrm{mV} / \mathrm{s})\end{array}$ & $\begin{array}{l}\text { Onset } \\
\text { potential }\end{array}$ & Current density & References \\
\hline $\begin{array}{l}\mathrm{Pd}_{4} \mathrm{Sn} \\
\text { nanowire }\end{array}$ & $0.1 \mathrm{M} \mathrm{KOH}$ & 10 & $\begin{array}{l}\sim 1.0 \mathrm{~V} \text { vs } \\
\text { RHE }\end{array}$ & $\begin{array}{l}0.65 \mathrm{~A} / \mathrm{mg}_{\mathrm{Pd}} \\
(@ 0.9 \mathrm{~V})\end{array}$ & $\begin{array}{l}\text { Nano Lett. } \\
\text { 2019, 19, } \\
6894-6903 \\
\end{array}$ \\
\hline $\begin{array}{l}\text { Co- } \\
\text { incorporated } \\
\mathrm{Pd}_{2} \mathrm{Sn} \\
\text { intermetallics }\end{array}$ & $0.5 \mathrm{M} \mathrm{KOH}$ & 10 & $\begin{array}{l}0.95 \mathrm{~V} \text { vs } \\
\text { RHE }\end{array}$ & $\begin{array}{l}0.1348 \mathrm{~A} / \mathrm{mgPd} \\
(@ 0.85 \mathrm{~V})\end{array}$ & $\begin{array}{l}\text { J. Electroanal. } \\
\text { Chem. 2017, } \\
789,167-163\end{array}$ \\
\hline $\begin{array}{l}\mathrm{PdSn} / \mathrm{C}(3: 1 \\
\text { molar ratio) }\end{array}$ & $\begin{array}{l}0.1 \mathrm{M} \\
\mathrm{HClO}_{4}\end{array}$ & 5 & $\begin{array}{l}\sim 0.6 \mathrm{~V} \mathrm{vs} \\
\mathrm{Ag} / \mathrm{AgCl}\end{array}$ & $\begin{array}{l}(0.55 \mathrm{~V}) \\
0.178 \mathrm{~mA} / \mathrm{cm}^{2}\end{array}$ & $\begin{array}{l}\text { ECS } \\
\text { Transactions } \\
\mathbf{2 0 1 4 , 6 4 ,} \\
97-106 \\
\end{array}$ \\
\hline $\begin{array}{l}\mathrm{PdSn} / \mathrm{C}(1: 1 \\
\text { molar ratio) }\end{array}$ & \multirow{2}{*}{$\begin{array}{l}0.1 \mathrm{M} \\
\mathrm{HClO}_{4}\end{array}$} & \multirow{2}{*}{20} & $\begin{array}{l}\sim 0.8 \mathrm{~V} \text { vs } \\
\text { RHE }\end{array}$ & $\begin{array}{l}(0.9 \mathrm{~V}) \\
0.0038 \mathrm{~A} / \mathrm{mg}_{\text {metal }} \\
0.0074 \mathrm{~A} / \mathrm{mg}_{\mathrm{Pd}}\end{array}$ & \multirow{2}{*}{$\begin{array}{l}\text { Fuel Cells } \\
\mathbf{2 0 1 0}, 10,734- \\
739\end{array}$} \\
\hline PdSn@Pt/C & & & $\begin{array}{l}\sim 1.0 \mathrm{~V} \text { vs } \\
\mathrm{RHE}\end{array}$ & $\begin{array}{l}(0.9 \mathrm{~V}) \\
0.0179 \mathrm{~A} / \mathrm{mg}_{\text {metal }} \\
\left(0.0613 \mathrm{~A} / \mathrm{mg}_{\mathrm{Pt}}\right)\end{array}$ & \\
\hline \multirow{2}{*}{$\begin{array}{l}\text { PdSn/rGO } \\
\text { hybrid } \\
\text { intermetallics }\end{array}$} & $\begin{array}{l}0.1 \mathrm{M} \\
\text { KOH }\end{array}$ & 50 & $\begin{array}{l}\text { 0.98 V vs } \\
\text { RHE }\end{array}$ & $\begin{array}{l}0.358 \mathrm{~A} / \mathbf{m g}_{\mathrm{Pd}} \\
(@ 0.82 \mathrm{~V})\end{array}$ & \multirow[b]{2}{*}{ This Work } \\
\hline & $\begin{array}{c}0.5 \mathrm{M} \\
\mathrm{H}_{2} \mathrm{SO}_{4}\end{array}$ & 5 & $\begin{array}{l}1.0 \mathrm{~V} \text { vs } \\
\text { RHE }\end{array}$ & $\begin{array}{l}0.187 \mathrm{~A} / \mathbf{m g}_{P d} \\
(@ 0.9 \mathrm{~V}) \\
0.424 \mathrm{~A} / \mathbf{m g}_{\mathbf{P d}} \\
(@ 0.88 \mathrm{~V})\end{array}$ & \\
\hline
\end{tabular}


Table S2. Pd-Pd interatomic distance and the electrocatalytic performances of the as-synthesized catalysts towards EGOR and ORR.

\begin{tabular}{|c|c|c|c|c|c|c|c|c|}
\hline \multirow{4}{*}{ Catalysts } & \multirow{4}{*}{$\begin{array}{l}\text { Pd-Pd } \\
\text { inter- } \\
\text { atomic } \\
\text { distance } \\
\text { in an } \\
\text { unit cell } \\
(\AA) *\end{array}$} & \multirow{4}{*}{$\begin{array}{c}\text { CO- } \\
\text { stripping } \\
\text { peak } \\
\text { potential } \\
(E @ C O) \\
(\mathrm{V} \text { vs } \\
\text { RHE) }\end{array}$} & \multicolumn{2}{|c|}{$\begin{array}{c}\text { EGOR } \\
\text { (in } 0.1 \mathrm{M} \mathrm{KOH})\end{array}$} & \multicolumn{2}{|c|}{$\begin{array}{c}\text { ORR } \\
\text { (in } 0.1 \mathrm{M} \mathrm{KOH})\end{array}$} & \multicolumn{2}{|c|}{$\begin{array}{c}\text { ORR } \\
\left(\text { in } 0.5 \mathrm{M} \mathrm{H}_{2} \mathrm{SO}_{4} \text { ) }\right.\end{array}$} \\
\hline & & & $\begin{array}{l}\text { Forward } \\
\text { peak }\end{array}$ & $\begin{array}{l}\text { Mass } \\
\text { activity }\end{array}$ & $\begin{array}{l}\text { Onset } \\
\text { potential }\end{array}$ & $\begin{array}{l}\text { Mass } \\
\text { activity }\end{array}$ & $\begin{array}{l}\text { Onset } \\
\text { potential }\end{array}$ & $\begin{array}{l}\text { Mass } \\
\text { l activity }\end{array}$ \\
\hline & & & potential & $\left(A / m g_{P d}\right)$ & & $\left(A / m g_{P d}\right)$ & & $\left(A / m g_{P d}\right)$ \\
\hline & & & RHE) & & RHE) & @0.82 V & RHE) & @0.88 V \\
\hline $\mathrm{Pd}_{3} \mathrm{Sn}_{2} / \mathrm{rGO}$ & 2.85 & 1.02 & 0.855 & 1.82 & 0.93 & 0.084 & 0.94 & 0.017 \\
\hline $\mathrm{PdSn} / \mathrm{rGO}$ & 3.06 & 0.99 & 0.848 & 4.34 & 0.98 & 0.358 & 1.0 & 0.424 \\
\hline $\mathrm{PdSn}_{2} / \mathrm{rGO}$ & 4.58 & 1.06 & 0.901 & 0.94 & 0.93 & 0.181 & 0.92 & 0.029 \\
\hline $\mathrm{Pd} / \mathrm{rGO}$ & 2.75 & 0.922 & 0.89 & 1.19 & 0.92 & 0.0172 & 0.93 & 0.018 \\
\hline
\end{tabular}

* Obtained from crystal structure database 